Pacific Journal of Mathematic 


\title{
CHARACTERISTIC SUBGROUPS OF MONOMIAL GROUPS
}

\author{
R. B. CROUCH
}

1. Introduction. Let $U$ be a set, $o(U)=B=\lambda^{\prime}{ }_{u}, u \geqq 0$, where $o(U)$ means the number of elements of $U$. Let $H$ be a fixed group. A monomial substitution $y$ is a transformation that maps every $x$ of $U$ in a one-to-one fashion into an $x$ of $U$ multiplied on the left by an element $h_{x}$ of $H$. Multiplication of substitutions means successive applications. The set of all monomial substitutions forms the monomial group $\Sigma$. Ore [5] has studied this group for finite $U$, and some of his results have been generalized to general $U$ in [2], [3], and [4].

This paper determines the structure of the characteristic subgroups for the case when $U$ is infinite in the cases where normal subgroups and automorphisms are known. The method used makes clear how corresponding theorems for the case where $U$ is finite might be proved but does not list these results.

2. Definitions and preliminaries. Let $d$ be the cardinal of the integers. Let $B$ be an infinite cardinal; $B^{+}$, the successor of $B ; U$, a set such that $o(U)=B$; and $C$ such that $d \leqq C \leqq B^{+}$. Let $H$ be a fixed group and $e$ the identity of $H$. Denote by $\Sigma=\Sigma(H ; B, d, C)$ the monomial group of $U$ over $H$ whose elements are of the form

$$
y=\left(\begin{array}{lr}
\cdots, & x_{\varepsilon}, \cdots \\
\cdots, h_{\varepsilon} x_{i_{\varepsilon}}, \cdots
\end{array}\right)
$$

where only a finite number of the $h_{\varepsilon}$ are not $e$ and the number of $x$ not mapped into themselves is less than $C$. Any element of $\Sigma$ may be written in the form

$$
y=\left(\begin{array}{lr}
\cdots, & x_{\varepsilon}, \cdots \\
\cdots, h_{\varepsilon} x_{\varepsilon}, \cdots
\end{array}\right)\left(\begin{array}{l}
\cdots, x_{\varepsilon}, \cdots \\
\cdots, \text { e } x_{i_{\varepsilon}}, \cdots
\end{array}\right)
$$

or $y=v s$ where $v$ sends every $x$ into itself and every $h$ of $s$ is $e$. Elements of the form of

$$
v=\left(\begin{array}{lr}
\cdots, & x_{\varepsilon}, \cdots \\
\cdots, h_{\varepsilon} x_{\varepsilon}, \cdots
\end{array}\right)=\left[\cdots, h_{\varepsilon}, \cdots\right]
$$

are multiplications and all such elements form a normal subgroup, the basis groups $V(B, d)=V$ of $\Sigma$. The $h_{\varepsilon}$ of $y$ are called the factors of $y$. Elements of the form of $s$ are permutations and all such elements form a subgroup, the permutation group, $S(B, C)=S$ of $\Sigma(H ; B, d, C)$. Cycles

Received March 6, 1959. 
of $s$ will also be written as $\left(x_{1}, \cdots, x_{n}\right)$ and $\left(\cdots, x_{-1}, x_{0}, x_{1}, \cdots\right)$. Baer [1] has shown that the normal subgroups of $S(B, C)$ are the alternating group, $A=A(B, d)$, and $S(B, D)$ where $d \leqq D \leqq C$. Let $E$ be the identity of $\Sigma, I$ the identity of $S$.

3. Characteristic subgroups of $\Sigma(H ; B, d, C), d \leqq C<B^{+}$. The normal subgroups of $\Sigma(H ; B, d, C)$ are known [2], [3]. They are classified first as to whether or not they are contained in the basis group $V$.

If $N$ is normal in $\Sigma$ and $N \subset V$ its elements are multiplications with only a finite number of non-identity factors which are contained in a normal subgroup $G$ of $H$. The set of all possible products of factors of all elements of $N$ form a normal subgroup $G_{1}$ of $H$. The group $G / G_{1}$ is Abelian and $G / G_{1}$ is in the center of $H / G_{1}$.

If $M$ is normal in $\Sigma$ and $M \not \subset V$ then $M \cap S=P \neq E$ is a normal subgroup of $S$. The group $N=M \cap V$ is as above except that $G=H$. It becomes necessary to consider the cases where $P=S(B, D)$ with $d \leqq D \leqq C$ and $P=A(B, d)$. When $P=S(B, D)$ then $M=N \cup P$.

If $M$ is normal in $\Sigma, M \not \subset V, P=A(B, d), M \cap V=N, M \mid N \cong A(B, d)$ then $M=N \cup A(B, d)$.

If $M$ is normal in $\Sigma, M \not \subset V, P=A(B, d), M \cap V=N, M / N \not \nexists A(B, \mathrm{~d})$ then $M=N \cup A(B, d) \cup L$ where $L$ is the cyclic group generated by $[e, a](1,2)$ with $a^{2} \in G_{1}, a \notin G_{1}$.

The converses of these theorems are true. That is, if one starts with the proper subgroups of $H$ and constructs $N$ or $M$ as above the resulting group is normal in $\Sigma$.

The automorphisms of $\Sigma(H ; B, d, C)$ are known [4]. A mapping $\theta$ is an automorphism of $\Sigma(H ; B, d, C)$ if and only if $\theta=T^{+} I_{\left(s^{+}\right)} I_{\left(v^{+}\right)}$where $T^{+}, I_{\left(s^{+}\right)}, I_{\left(v^{+}\right)}$are automorphisms of $\Sigma$ defined as follows. Let $T$ be any automorphism of $H$. Then

$$
y T^{+}=v s t^{+}=\left[h_{1}, \cdots, h_{\varepsilon}, \cdots\right] s T^{+}=\left[h_{1}^{T}, \cdots, h_{\varepsilon}^{T}, \cdots\right] s .
$$

Let $s^{+} \in S\left(B, B^{+}\right)$. Then $I_{\left(s^{+}\right)}$is defined by $y I_{\left(s^{+}\right)}=s^{+} y\left(s^{+}\right)^{-1}$. Let $v^{+} \in$ $V\left(B, B^{+}\right)$if $C=d, v^{+} \in V(B, d)$ if $d<C$ then $I_{\left(v^{+}\right)}$is defined by $y I_{\left(v^{+}\right)}=$ $v^{+} y\left(v^{+}\right)^{-1}$.

Theorem 1. If $N$ is a subgroup of $\Sigma(H ; B, d, C)$ contained in the basis group then $N$ is characteristic in $\Sigma$ if and only if $N$ is normal in $\Sigma$, (hence is as described above) and $G, G_{1}$ are characteristic in $H$.

Proof. Assume $N$ is characteristic in $\Sigma$. Then $N$ is normal in $\Sigma$ and its structure is known. Choose $\theta=T^{+}$with $T$ arbitrary in the automorphism group of $H$ and $v$ arbitrary in $N$. Then 


$$
\begin{aligned}
v \theta & =\left[e, \cdots, e, e, g_{i_{1}}, e, \cdots, e, g_{i_{n}}, e, \cdots\right] T^{+} \\
& =\left[e, \cdots, g_{i_{1}}^{T}, e, \cdots, e, g_{i_{n}}^{T}, e, \cdots\right]
\end{aligned}
$$

The elements $g_{i_{1}}^{T}$ must be in $G$. This shows $G$ is characteristic in $H$. Furthermore $g_{i_{1}}^{T} g_{i_{2}}^{T} \cdots g_{i_{n}}^{T}=\left(g_{i_{1}} \cdots g_{i_{n}}\right)^{T}$ must be in $G_{1}$ and since $g_{i_{1}} \cdots g_{i_{n}}$ is arbitrary in $G_{1}, G_{1}$ is characteristic in $H$.

Conversely, if $N \subset V(B, d), N$ is normal in $\Sigma, G, G_{1}$ are characteristic in $H$ then $N$ is characteristic in $\Sigma$. To see this let $v_{1}$ be arbitrary in $N$. Then $v_{1} \theta=v_{1} T I_{\left(s^{+}\right)} I_{\left(v^{+}\right)}=v_{2} I_{\left(s^{+}\right)} I_{\left(v^{+}\right)}$. The non-identity factors of $v_{2}$ are in $G$ and their product in $G_{1}$ by $G, G_{1}$ characteristic in $H$. Now $v_{2} I_{\left(s^{+}\right)} I_{\left(v^{+}\right)}=$ $\left(v^{+}\right)\left(s^{+}\right) v_{2}\left(s^{+}\right)^{-1}\left(v^{+}\right)^{-1}$. The effect of $I_{\left(s^{+}\right)}$on $v_{2}$ is to permute the nonidentity factors so $\left(v^{+}\right)\left(v_{3}\right)\left(v^{+}\right)^{-1}$ is now to be considered with $v_{3}$ in $N$. Since $G$ is normal in $H$ in $G / G_{1}$ is in the center of $H / G_{1},\left(v^{+}\right) v_{3}\left(v^{+}\right)^{-1}$ will be in $N$.

THeORem 2. Let $M=N \cup P$ be a normal subgroup of $\Sigma(H ; B, d, C)$, $d \leqq C<B^{+}$, where $N$ is as described above, $P=S(B, D)$. Then $M$ is characteristic in $\Sigma$ if and only if $G_{1}$ is characteristic in $H$.

Proof. By an argument similar to that used in Theorem 1, $G_{1}$ is characteristic in $H$.

Conversely, if $y=v_{1} s_{1}$ is arbitrary in $M$ then

$$
v_{1} s_{1} \theta=v_{1} s_{1} T^{+} I_{\left(s^{+}\right)} I_{\left(v^{+}\right)}=v_{2} s_{1} I_{\left(s^{+}\right)} I_{\left(v^{+}\right)} .
$$

Since $G_{1}$ is characteristic in $H, v_{2}$ belongs to $N$. Now consider

$$
\left(v^{+}\right)\left(s^{+}\right) v_{2} s_{1}\left(s^{+}\right)^{-1}\left(v^{+}\right)^{-1}=\left(v^{+}\right) v_{3}\left(s^{+}\right) s_{1}\left(s^{+}\right)^{-1}\left(v^{+}\right)^{-1}=\left(v^{+}\right) v_{3} s_{2}\left(v^{+}\right)^{-1} .
$$

The multiplication $v_{3}$ is in $N$ since the factors are still in $H$, and the product of the factors is still in $G_{1}$ since $H / G_{1}$ is Abelian. The permutation $s_{2}$ is in $P$ since $P$ is normal in $S\left(B, B^{+}\right)$. It is now convenient to consider two cases. If $C=d$ the permutation $s_{2}$ is finite and $\left(v^{+}\right) v_{3} s_{2}\left(v^{+}\right)^{-1}=$ $\left(v^{+}\right) v_{3} v_{4} s_{2}$ where the factors of $v_{4}$ differ from the inverse of those in $\left(v^{+}\right)$ in only a finite number of places. Therefore $\left(v^{+}\right) v_{3} v_{4}$ will have a finite number of factors of the form $k_{\varepsilon} h_{\varepsilon} k_{i_{\varepsilon}}^{-1}$. If $k_{\varepsilon} \neq k_{i_{\varepsilon}}$ then $k_{i_{\varepsilon}} h_{i_{\varepsilon}} k_{a}, k_{i \varepsilon} \neq k_{a}$, will be a factor of $(v) v_{3} v_{4}$. Since $H / G_{1}$ is Abelian the product of the factors is in $G_{1}$. Therefore, $\left(v^{+}\right) v_{3} v_{4} s_{2}=v_{5} s_{2}$ belongs to $M$. If $C>d$ then $\left(v^{+}\right)$, $v_{4}$ have only a finite number of non-identity factors and the same argument holds. Therefore $\left(v^{+}\right) v_{3} v_{4} s_{2}$ belongs to $M$.

THEOREM 3. Let $M=N \cup A(B, d)$ be a normal subgroup of $\Sigma(H ; B, d, C)$, $d \leqq C<B^{+}$. Then $M$ is characteristic in $\Sigma$ if and only if $G_{1}$ is characteristic in $H$. 
Proof. The argument used in the proof of Theorem 1 may be used to show that $G_{1}$ is characteristic in $H$ if $M$ is characteristic in $\Sigma$.

Conversely, if $y=v_{1} s_{1}$ is arbitrary in $M$ then

$$
\begin{aligned}
y \theta=v_{1} s_{1} \theta & =v_{1} s_{1} T^{+} I_{\left(s^{+}\right)} I_{\left(v^{+}\right)}=v_{2} s_{1} I_{\left(s^{+}\right)} I_{\left(v^{+}\right)}=\left(v^{+}\right)\left(s^{+}\right) v_{2} s_{1}\left(s^{+}\right)^{-1}\left(v^{+}\right)^{-1} \\
& =\left(v^{+}\right) v_{3}\left(s^{+}\right) s_{1}\left(s^{+}\right)^{-1}\left(v^{+}\right)^{-1}=\left(v^{+}\right) v_{3} s_{2}\left(v^{+}\right)^{-1}=\left(v^{+}\right) v_{3} v_{4} s_{2} .
\end{aligned}
$$

Now $v_{2} \in N$ by $G_{1}$ characteristic in $H$ and $v_{3}$ will be in $N$ by $H / G_{1}$ Abelian. Since $A(B, d)$ is normal in $S\left(B, B^{+}\right), s_{2}$ belongs to $A(B, d)$. The factors of $v_{4}$ differ from the inverse of those in $v$ in only a finite number of places since $s_{2}$ moves only a finite number of $x$. Therefore, $\left(v^{+}\right) v_{3} v_{4} \in N, s_{2} \in A(B, d)$ and $M$ is characteristic in $\Sigma$.

THEOREM 4. Let $M_{1}=N \cup A \cup L$ be a normal subgroup of $\Sigma(H$; $B, d, C), d \leqq C<B^{+}$. Let $L$ be generated by $y=[e, a](1,2)$ with $a^{2} \in G_{1}$, $a \notin G_{1}$. Then $M_{1}$ is characteristic in $\Sigma$ if and only if $G_{1}$ is characteristic in $H$, and $a^{T}$ belongs to the coset $a G_{1}$ for all automorphisms $T$ of $H$.

Proof. By considering $v \in N$ and $\theta=T^{+}$we see that $G_{1}$ is characteristic in $H$. By considering $y=[e, a](1,2)$ of $M_{1}$ and $\theta=T^{+}$we see that $\left[e, a^{T}\right](1,2)$ must belong to $M_{1}$. This means $a^{T}$ belongs to $a G$.

Conversely, if $v_{1} s_{1} \in M_{1}$ then

$$
\begin{aligned}
v_{1} s_{1} \theta & =v_{1} s_{1} T^{+} I_{\left(s^{+}\right)} I_{\left(v^{+}\right)}=v_{2} s_{1} I_{\left(s^{+}\right)} I_{\left(v^{+}\right)}=\left(v^{+}\right)\left(s^{+}\right) v_{2} s_{1}\left(s^{+}\right)^{-1}\left(v^{+}\right)^{-1} \\
& =\left(v^{+}\right) v_{3}\left(s^{+}\right) s_{1}\left(s^{+}\right)^{-1}\left(v^{+}\right)^{-1}=\left(v^{+}\right) v_{3} s_{2}\left(v^{+}\right)^{-1}=\left(v^{+}\right) v_{3} v_{4} s_{2} .
\end{aligned}
$$

Now $v_{2} s_{1}$ is in $M_{1}$ by $G_{1}$ characteristic if the product of the factors of $v_{1}$ is in $G_{1}$ and by $a^{T}$ in $a G_{1}$ if the product of the factors is in $a G_{1}$. The multiplication $v_{3}$ has only a finite number of non-identity factors because $v_{2}$ has only a finite number of non-identity factors. Since $s_{1}$ is finite, $s_{2}$ is a finite permutation and is even or odd as $s_{1}$ is even or odd. Therefore, $v_{4}$ has only a finite number of factors different from the inverse of the factors of $\left(v^{+}\right)$. The factors of $\left(v^{+}\right) v_{3} v_{4}$ have their product in $G_{1}$ or $a G_{1}$ according as $v_{3}$ has its product in $G_{1}$ or $a G_{1}$. Therefore, if $s_{1}$ was even $s_{2}$ is even, $v_{1}$ had the product of its factors in $G_{1}$ and so does $\left(v^{+}\right) v_{3} v_{4}$. If $s_{1}$ was odd so is $s_{2}$ and $v_{1}$ had the product of its factors in $a G_{1}$ and so does $\left(v^{+}\right) v_{3} v_{4}$. That is, $M_{1}$ is characteristic.

4. Characteristic subgroups of $\Sigma_{A}(H ; B, d, d)$. The normal subgroups of $\Sigma_{A}(H ; B, d, d)$ are precisely those of $\Sigma(H ; B, d, d)$ that are contained in $\Sigma_{A}(H ; B, d, d)[2, \mathrm{p} .210]$. The automorphism of $\Sigma_{A}(H ; B, d, d)$ are those of $\Sigma(H ; B, d, d)$ restricted to $\Sigma(H ; B, d, d)[4$, p. 84]. 
THEOREM 5. Let $N$ be a subgroup of $\Sigma_{A}(H ; B, d, d)$ contained in the basis group. Then $N$ is characteristic in $\Sigma_{A}$ if and only if $N$ is normal in $\Sigma_{A}$ and $G, G_{1}$ are characteristic in $H$.

THeOREM 6. Let $M$ be a subgroup of $\Sigma_{A}(H ; B, d, d), M \not \subset V(B, d)$. Then $M$ is characteristic in $\Sigma_{A}$ if and only if $M$ is normal, i.e. $M=$ $N \cup A$, and $G_{1}$ is characteristic in $H$.

\section{BIBLIOGRAPHY}

1. R. Baer, Die Kompositionsreihe der Gruppe aller einendeutigen Abbildungen einer unendlichen Menge auf sich, Studia Mathematica, 5 (1934), 15-17.

2. R. B. Crouch, Monomial Groups, Trans. Amer. Math. Soc., 80 (1955), 187-215.

3. R. B. Crouch and W. R. Scott, Normal subgroups of monomial groups, Proc. Amer. Math. Soc., 8, No. 5 (1957), 931-936.

4. C. V. Holmes, Contributions to the Theory of Groups, Research Grant NSF-G 1126, Report No. 5, Feb., (1956), 23-93.

5. O. Ore, Theory of monomial groups, Trans. Amer. Math. Soc., 51 (1942), 15-64.

NeW MeXico State University

University PARK, NeW Mexico 



\title{
PACIFIC JOURNAL OF MATHEMATICS
}

\section{EDITORS}

\author{
David Gilbarg \\ itanford University \\ itanford, California \\ F. H. Brownele \\ Jniversity of Washington \\ ieattle 5 , Washington
}

\author{
A. L. Whiteman
}

University of Southern California Los Angeles 7. California

L. J. Paige

University of California

Los Angeles 24, California

ASSOCIATE EDITORS
ミ. F. BECKENBACH
E. HEWITT
M. OHTSUKA
E. SPANIER
r. M. CHERRY
A. HORN
H. L. ROYDEN
E. G. STRAUS
). DERRY
L. NACHBIN
M. M. SCHIFFER
F. WOLF

\section{SUPPORTING INSTITUTIONS}

JNIVERSITY OF BRITISH COLUMBIA
ZALIFORNIA INSTITUTE OF TECHNOLOGY
JNIVERSITY OF CALIFORNIA
UONTANA STATE UNIVERSITY
JNIVERSITY OF NEVADA
NEW MEXICO STATE UNIVERSITY
JREGON STATE COLLEGE
JNIVERSITY OF OREGON
JSAKA UNIVERSITY
JNIVERSITY OF SOUTHERN CALIFORNIA

STANFORD UNIVERSITY

UNIVERSITY OF TOKYO

UNIVERSITY OF UTAH

WASHINGTON STATE COLLEGE

UNIVERSITY OF WASHINGTON

AMERICAN MATHEMATICAL SOCIETY CALIFORNIA RESEARCH CORPORATION HUGHES AIRCRAFT COMPANY

SPACE TECHNOLOGY LABORATORIES

NAVAL ORDNANCE TEST STATION

Printed in Japan by Kokusai Bunken Insatsusha

(International Academic Printing Co., Ltd.), Tokyo, Japan 


\section{Pacific Journal of Mathematics}

\section{Vol. 10, No. $1 \quad$ September, 1960}

Richard Arens, Extensions of Banach algebras................... 1

Fred Guenther Brauer, Spectral theory for linear systems of differential equations....................................... 17

Herbert Busemann and Ernst Gabor Straus, Area and normality ......... 35

J. H. Case and Richard Eliot Chamberlin, Characterizations of tree-like

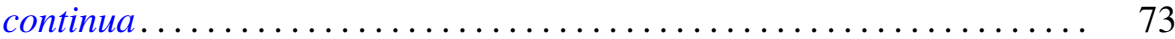

Ralph Boyett Crouch, Characteristic subgroups of monomial groups...... 85

Richard J. Driscoll, Existence theorems for certain classes of two-point boundary problems by variational methods ................. 91

A. M. Duguid, A class of hyper-FC-groups ................. 117

Adriano Mario Garsia, The calculation of conformal parameters for some

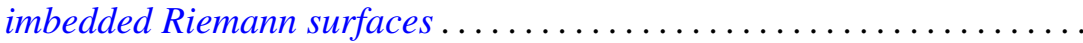

Irving Leonard Glicksberg, Homomorphisms of certain algebras of measures ........................................... 167

Branko Grünbaum, Some applications of expansion constants . . . . . . . . . 193

John Hilzman, Error bounds for an approximate solution to the Volterra integral equation ................................... 203

Charles Ray Hobby, The Frattini subgroup of a p-group ............... 209

Milton Lees, von Newmann difference approximation to hyperbolic

equations...................................... 213

Azriel Lévy, Axiom schemata of strong infinity in axiomatic set theory .... 223

Benjamin Muckenhoupt, On certain singular integrals ............... 239

Kotaro Oikawa, On the stability of boundary components ............. 263

J. Marshall Osborn, Loops with the weak inverse property .............. 295

Paulo Ribenboim, Un théorème de réalisation de groupes réticulés ....... 305

Daniel Saltz, An inversion theorem for Laplace-Stieltjes transforms ....... 309

Berthold Schweizer and Abe Sklar, Statistical metric spaces ........... 313

Morris Weisfeld, On derivations in division rings ................ 335

Bertram Yood, Faithful ${ }^{*}$-representations of normed algebras ........... 345 